\title{
An Overview of the DARPA Autonomous Robotic Manipulation (ARM) Program
}

\author{
Douglas Hackett*1, James Pippine ${ }^{* 2}$, Adam Watson*2, Charles Sullivan*3 and Gill Pratt*4 \\ ${ }^{* 1}$ Griffin Technologies ${ }^{* 2}$ System Planning Corporation ${ }^{* 3}$ Golden Knight Technologies ${ }^{* 4}$ DARPA Defense Sciences Office
}

\section{Introduction}

Circa 2010, manipulation systems required burdensome human operation and high-precision arms and hands, yet could not match the manipulation skills of a two-year old child. To address this situation, the U.S. Defense Advanced Research Projects Agency (DARPA) began funding the Autonomous Robotic Manipulation (ARM) program. The program's goalsare to develop intelligent control of manipulators that perform subtasks on their own, and to reduce the cost of robot hardware for wider adoption.

The ARM program comprises four tracks: software, low-cost hand, low-cost arm, and outreach. ARM-S, the software track, is developing new techniques for grasping and manipulation using local sensors for perception. ARM-H, the low-cost hand track, focused on design and development of low-cost dexterous, multi-fingered robot hands. The low-cost arm track is addressing a lowcost, dual-use robot/prosthetic application. The outreach track is providing the public with an opportunity to interact with the ARM technology. This paper describes recent work in both ARM-S and ARM-H.

\section{The ARM-S Track}

Developing and testing autonomous systems has traditionally presented a number of challenges. Re-

原稿受付 2013 年 1 月 29 日

キーワード : Autonomous Manipulation, Manipulators, Sensing, Evaluation

*1 802 Montgomery Avenue, Fort Washington, PA 19034, USA

*23601 Wilson Blvd, Arlington, VA 22201, USA

*313122 Moss Ranch Lane, Fairfax, VA 22033-3524, USA

${ }^{* 4} 675$ North Randolph Street, Arlington, VA 22203-2114, USA

The views, opinions, and/or findings contained in this article/presentation are those of the author/ presenter and should not be interpreted as representing the official views or policies, either expressed or implied, of the Defense Advanced Research Projects Agency or the Department of Defense. searchers often lack sufficiently capable hardware and can struggle with maintaining it. Results of algorithms run on different platforms and using different test sets can be difficult to compare and benchmark. Evaluating these algorithms often comprises an extensive, complex event at the end of a long development phase.

This process does not allow iterative improvement or assessment of progress throughout the program. DARPA has directly addressed these limitations on the ARM Program. Teams are supplied with identical robot hardware, a regular test structure, and a variety of test challenges.

The ARM-S track comprises two phases of 17 and 21 months. Six teams were initially funded based on their expertise and novel approaches: Carnegie Mellon University, HRL Laboratories, iRobot, NASA Jet Propulsion Laboratory, SRI International, and the University of Southern California. Phase 1 was completed in November, 2011, and Phase 2 is in progress through 2013.

Robot In previous DARPA robotics programs, research teams would be responsible for building and maintaining a robot, writing low-level operating system code to run it, and then developing autonomous software to address the research objectives. This approach diluted the research effort and madecomparisons of test results very difficult. To avoid these pitfalls, DARPA commissioned $\mathrm{re}^{2}[1]$ of Pittsburgh, PA to build a robot for the program, which was furnished to all of the ARM$\mathrm{S}$ teams.

The ARM robot (Fig. 1) integrates state-of-the-art hands, arms, and perception sensors [2]. It is controlled by a pair of desktop workstations. One controls the arms and hands using a real-time operating system and CAN bus connection. The other hosts the team's software for sensing, planning, and user interface.

In Phase 1 the teams were provided with one arm and 


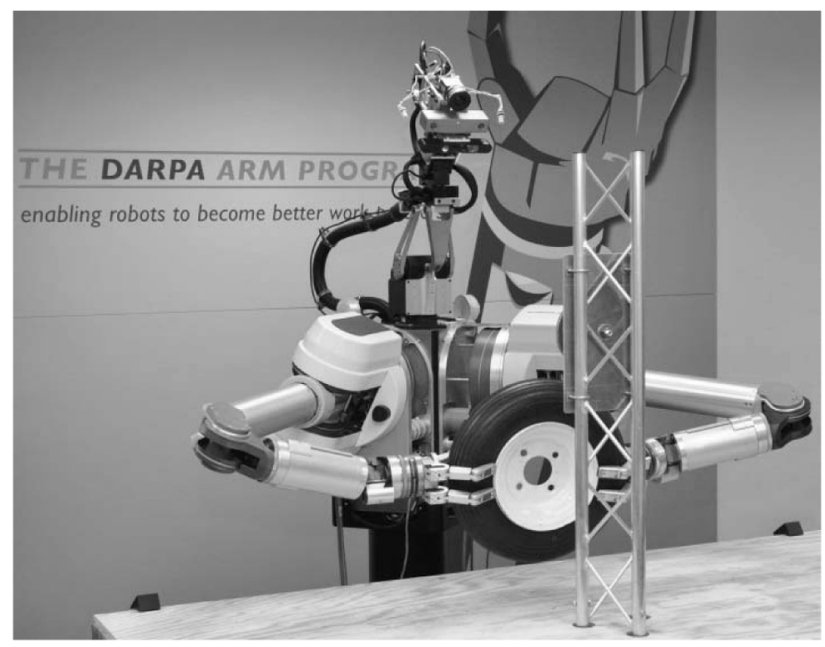

Fig. 1 The ARM Robot, with covers removed

one hand, while in Phase 2 teams are using two arms and hands for bimanual tasks. Developers are permitted to use any software environment, libraries, or tools that they need. Many teams build on ROS and add their own custom software.

Evaluation An important part of the program is regular, independent testing. Performer teams develop and test software at their own sites on their ARM robot. At roughly six-week intervals, teams upload their software to the DARPA test site, where the test team performs a series of independent spiral tests using a separate copy of the robot. These intermediate trials are for practice but at the end of each phase, a final, formal evaluation is conducted.

During each intermediate test, teams held a multimedia teleconference with the test site, where they could see the robot perform and observe any software diagnostics while discussing the results with the test team. Both teams gained a richer understanding of how the autonomy performed.

This type of testing has some key advantages. Both the teams and DARPA receive regular feedback on the research progress. The difficulty of the tests can be modified to explore robustness and to push the teams' performance. Finally, the tests can focus on real-world problems.

Each ARM-S test consisted of a series of grasping or manipulation challenge tasks. For grasping tasks, an object was placed in one of five different poses on a table in front of the robot. The human indicated what object type was to be grasped, and the robot's task was to perceive and locate the object, and then pick it up.



Fig. 2 Some Phase 1 Challenges - Grasping (top) and Manipulation (bottom)

To ensure that the grasp was firm, the robot then placed the object in a designated region on the table. Manipulation challenges were similar - only high-level direction was given (e.g., 'open the door'). Success was defined as completion of the task, and each trial was timed. Some example tasks are illustrated in Fig. 2.

The objects to be grasped included a ball, shovel, flashlight, floodlight bulb, radio, hammer, rock screwdriver, and case. The manipulation challenges were actuating a stapler, turning on a flashlight, hanging up a telephone, opening a door, unlocking a door, and drilling a hole.

Because perception was a necessary element but not a focus of the program, the teams were given a 3-D model of each object prior to testing. Additional trials were conducted with a novel hammer, rock, and screwdriver that had no specific scan file. The unseen objects were of the same general form as the scanned object, but of different dimensions than what the teams had practiced on.

The primary ARM-S evaluation criterion was success (binary) in completing the task. The best four out of five runs were used for computing scores and statistics. In case of ties, the secondary criterion was the average time of the trials.

Phase 1 Results A final evaluation was held at the end of ARM-S Phase 1 in November, 2011. Table 1 lists the results from the six teams.

The top three of the six teams (A, B, and C) were 
Table 1 ARM-S Test Results-Successes

\begin{tabular}{ccccc}
\hline Team & $\begin{array}{c}\text { Successes } \\
(\leq 72)\end{array}$ & $\begin{array}{c}\text { Grasping } \\
(\leq 48)\end{array}$ & $\begin{array}{c}\text { Manipulation } \\
(\leq 24)\end{array}$ & $\begin{array}{c}\text { Avg. Time } \\
(s)\end{array}$ \\
\hline $\mathrm{A}$ & 67 & 47 & 20 & 75.4 \\
\hline $\mathrm{B}$ & 67 & 47 & 20 & 80.6 \\
\hline $\mathrm{C}$ & 64 & 46 & 18 & 77.5 \\
\hline $\mathrm{D}$ & 58 & 47 & 11 & 125.7 \\
\hline $\mathrm{E}$ & 58 & 41 & 17 & 170.7 \\
\hline $\mathrm{F}$ & 49 & 42 & 7 & 151.8 \\
\hline
\end{tabular}

selected to move into Phase 2. The second phase focuses on using two arms and hands to perform a set of extended, real-world scenarios. One of these scenarios, changing a tire, is shown in Fig. 1.

\section{The ARM-H Track}

Regardless of how good the ARM-S technology becomes, it will be very difficult to transition to military or commercial applications if it requires the use of $\$ 150,000$ USD manipulators. Therefore, the goal of the ARM hardware track was to design and build a rugged, dexterous, multi-fingered hand with an order of magnitude lower cost than existing designs. This would make it possible to replace currently fielded claw-like manipulators with hands having 3-4 fingers and a useable palm.

Three teams were funded for the two-year program: iRobot, Sandia National Laboratories, and SRI International. All three successfully passed through the critical design review in 2011 and proceeded to a full evaluation in June, 2012. The three completed hands are shown in Fig. 3.

Evaluation To test the ability of the newlydeveloped hands to perform manipulation tasks, a formal evaluation was held at the DARPA test site. Each team performed a set of grasping and manipulation challenges that were very similar to those in ARM-S. Tasks included picking up objects such as a canteen, floodlight or drill, and performing manipulation such as putting a battery in a flashlight or turning a valve. Each team also attempted to pick up a $6.8[\mathrm{~kg}]$ dumbbell as a test of hand strength (For this particular test, the hand gripped the object and was manually lifted at the wrist).

For these trials the hand was mechanically attached to the ARM robot, but electrically connected to the teams' own controller. The ARM robot's arm was connected to a Barrett 7-AXIS Gimbals/Master Arm Sys-

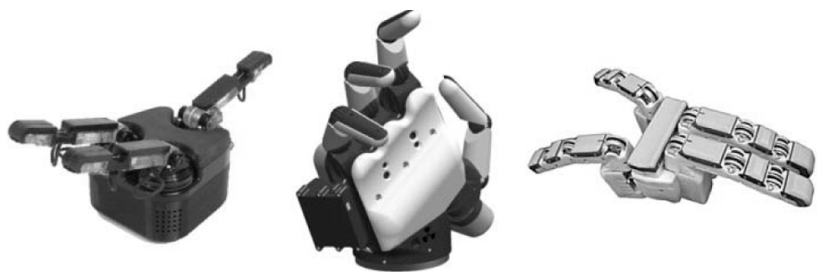

Fig. 3 The ARM-H Hands-iRobot (left), Sandia (middle), and SRI (right)



Fig. 4 ARM-H Testing in Progress

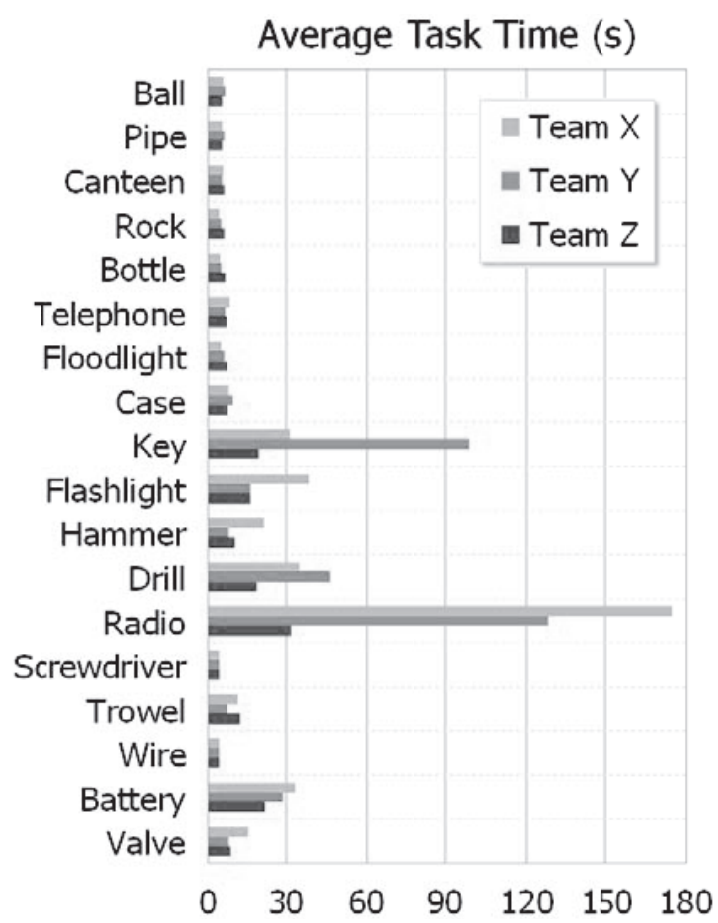

Fig. 5 ARM-H Test Results-Run Time

tem, which allowed one team member to teleoperate the robot's arm. The other person controlled the motion of the hand. Therefore, trials used two operators, directly viewing the scene, working in tandem. Fig. 4 illustrates the ARM-H test configuration.

The evaluation was conducted with a protocol very similar to ARM-S: the object was presented in different poses for five timed trials. The primary metric was 
Table 2 ARM-H Test Results-Successes

\begin{tabular}{ccccc}
\hline Team & $\begin{array}{c}\text { Grasping } \\
(\leq 48)\end{array}$ & $\begin{array}{c}\text { Manipulation } \\
(\leq 24)\end{array}$ & $\begin{array}{c}\text { Strength } \\
(\leq 2)\end{array}$ & $\begin{array}{c}\text { Total } \\
(\leq 74)\end{array}$ \\
\hline $\mathrm{X}$ & 56 & 13 & 2 & 71 \\
\hline $\mathrm{Y}$ & 56 & 16 & 2 & 74 \\
\hline $\mathrm{Z}$ & 52 & 16 & 2 & 74 \\
\hline
\end{tabular}

task completion, and the secondary metric was average task time. The top four out of five runs were used for statistics.

Like ARM-S, each of the challenge tasks was performed five times, in different poses. The primary evaluation criterion was success in completing the task. In case of ties, the secondary criterion was time required. The best four out of five runs were used for computing scores and statistics.

Results All teams were able to complete each of the



\section{Douglas D. Hackett}

Mr. Hackett is a Principal at Griffin Technologies, managing research in robotics, machine perception, and human augmentation. He serves as the Test Director for the ARM program. Before joining Griffin, Mr. Hackett worked in industry at biological simulation, medical device, and defense companies. Mr. Hackett has Master's and Bachelor's degrees in Electrical Engineering from the University of Illinois and Carnegie Mellon University, respectively.

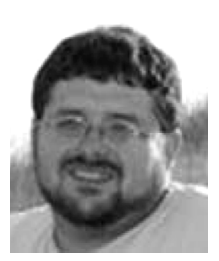

\section{Adam Watson}

Mr. Watson is a Systems Engineer at System Planning Corporation, focused on testing and evaluating robotics systems. He serves as the technical test lead for the ARM program. Mr. Watson has a Master's degree in Electrical Engineering from North Carolina State University and a Bachelor's in Computer Engineering from the University of North Carolina.

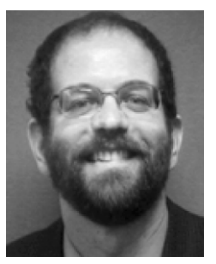

\section{Gill A. Pratt}

Dr. Gill Pratt is a Program Manager in the DARPA Defense Sciences Office, and runs the ARM Program. Dr. Pratt's primary interest is in the field of robotics: declarative design methods that enhance the symbiosis between designer and design tool, rapid fabrication methods, interfaces that enhance human/machine collaboration, mechanisms and control methods for enhanced mobility and manipulation, innovative actuators, and the application of neuroscience techniques to robot perception and control. Dr. Pratt holds a doctorate in electrical engineering and computer science from the Massachusetts Institute of Technology (MIT). Before DARPA, he was the Associate Dean of Faculty Affairs and Research at Franklin W. Olin College. challenge tasks. Table 2 lists the successes by team, and Fig. 5 illustrates the average task time by object. The top team (X) has been funded to manufacture multiple hands to the ongoing ARM-S track, where they will be used extensively in real-world scenarios.

\section{Conclusion and Future Work}

To date, the ARM performer teams have performed beyond expectations, producing three outstanding robot hands and making important advances in robust software performance. We believe that this effort has raised the state of the art in robotic manipulation and look forward to even more success in the remainder of the program.

\section{References}

[1] http://www.resquared.com/

[2] http://thearmrobot.com/index.html



\section{James Pippine}

Mr. Pippine is President of Golden Knight Technologies, focusing on research and development of robotics, autonomous systems and advanced platforms. He serves as the lead technical and programmatic consultant to the ARM program. Mr. Pippine holds a degree in Chemical Engineering from the Clarkson University in Potsdam, New York.

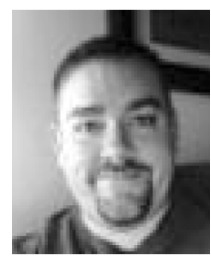

\section{Charles Sullivan}

Mr. Sullivan is a Mechanical Engineer at System Planning Corporation. He serves as a Test Engineer on the ARM program. Mr. Sullivan holds a degree in Mechanical Engineering from the University of Maryland, College Park. 